\title{
The masking of octave-band noise by broad-spectrum noise: A comparison of infant and adult thresholds
}

\author{
DALE BULL, BRUCE A. SCHNEIDER, and SANDRA E. TREHUB \\ Centre for Research in Human Development \\ Erindale College, University of Toronto, Mississauga, Ontario L5L 1C6, Canada
}

\begin{abstract}
Localization responses to a 4,000-Hz octave-band noise in a background of broad-spectrum noise were obtained from infants, 6, 12, 18, and 24 months of age, and adults. A twoalternative, forced-choice procedure was used to determine thresholds at each of two levels of masking noise, 42 and $60 \mathrm{dBC}$. Adults were also tested for their localization of pure tones in noise and their detection of octave-band noises with the more traditional two-interval, forced-choice task. Increasing the masking noise from 42 to $60 \mathrm{dBC}$ resulted in comparable threshold shifts for all age groups. However, infant thresholds were 16-25 dB higher than those obtained for adults. The theoretical implications of these findings are discussed.
\end{abstract}

Although the detection of signals in noise has been studied extensively in adult populations, little is known about auditory masking in infants and very young children. Mills (1975) has noted that children require greater signal-to-noise ratios than adults in various speech-intelligibility tasks, but such tasks obviously preclude application to infants and, moreover, involve cognitive as well as sensory factors. Elliott and Katz (1980) have reported that the introduction of background noise raises thresholds for a 2,000-Hz tone in a similar fashion for 6-year-old children and adults, but their three-alternative, forced-choice technique with buttonpress is inappropriate for infants and very young children.

Since Moore, Wilson, and Thompson (1977) demonstrated that the auditory-localization response could be reinforced in infants as young as 5 months of age, the response has been used to ascertain absolute thresholds in quiet backgrounds for infants 524 months of age (Moore \& Wilson, 1978; Schneider, Trehub, \& Bull, 1980; Trehub, Schneider, \& Endman, 1980). The purpose of the present investigation was to extend these procedures to the study of masking in infancy. In contrast with Moore et al. (1977), who used a single loudspeaker and fixed response interval, we used two loudspeakers and no-limit, forced-choice responding. The advantages of these modifications are outlined in Trehub, Schneider, and Bull (1981) and Trehub et al. (1980).

Pilot testing revealed that infants had difficulty

This research was supported by grants from the Medical Research Council of Canada and the University of Toronto. Requests for reprints should be sent to B. A. Schneider, Centre for Research in Human Development, Erindale College, University of Toronto, Mississauga, Ontario L5L 1C6, Canada. localizing sine waves but no such difficulty with band noises. Accordingly, the detection of an octave-band noise centered at $4,000 \mathrm{~Hz}$ in a background of broadspectrum noise was determined for infants $6,12,18$, and 24 months of age as well as for adults. Since studies of adult thresholds typically involve the detection of tones in noise using different psychophysical techniques, two additional questions were addressed with adults. First, masked thresholds for $4,000-\mathrm{Hz}$ tones were determined using the same localization technique employed for the infants. Second, thresholds in quiet were determined for $4,000-\mathrm{Hz}$ octaveband noise using both the localization task and the more traditional two-interval, forced-choice task.

\section{METHOD}

\section{Subjects}

The subjects were 134 full-term infants at $6,12,18$, or 24 months of age, all free of colds on the test day. Each infant was tested individually at two levels of masking noise. The infants were excluded from the final sample for failing to reach the training criterion $(n=5)$, failing to complete all trials at one masking level because of fussing or crying $(n=15)$, or for equipment problems $(n=6)$. The final sample of 108 infants comprised 29 infants at 6 months of age (mean $=26.7$ weeks), 31 at 12 months of age (mean $=52.9$ weeks), 28 at 18 months of age (mean $=79.2$ weeks), and 20 at 24 months of age (mean $=104.3$ weeks). In each age group, at least 19 infants completed all trials at both masking levels. A total of $\mathbf{4 0}$ adults (mean age $=\mathbf{2 2}$ years) were also tested.

\section{Apparatus and Stimuli}

A circuit diagram of the apparatus is presented in Figure 1. The 4,000-Hz octave-band noise was produced by filtering the output of a noise generator (General Radio, Model 1391) with a filter (General Radio 1952) set to pass an octave band centered at $4,000 \mathrm{~Hz}$. The filter output was directed to two electronic switches (Grason-Stadler 1287B), and when a trial was initiated, relay circuitry activated one or the other switch. The output of either switch (rise/decay time $=25 \mathrm{msec}$ ) was passed through an attenu- 




Figure 1. Circuit diagram for the presentation of filtered noise in a background of broad-spectrum noise.

ator (Hewlett-Packard 350D) to an input channel of one of a pair of differential amplifiers (Tektronix AM502). For the background masker, the output of a second noise generator was led through two parallel circuits, each circuit consisting of an electronic switch and an attenuator. Each circuit drove the second input of one of the differential amplifiers. During an experimental session, the electronic switches controlling the masking noise were always on. The output of the two differential amplifiers then served as input to a stereo amplifier (Technics, Model SU7300), one output to the left channel and the other to the right channel. Thus, with the signal switched off, the masking noise was presented over two loudspeakers (ESS-Heil, Model AMT1AM) connected to the stereo amplifier. When a trial was initiated, the signal plus noise was presented over the appropriate speaker with no attendant changes in the state of the other speaker.

The speakers were placed in a double-walled sound-attenuating chamber (Industrial Acoustics) $1.85 \mathrm{~m}$ away from the center of a chair that occupied one corner of the room. One speaker was located $45 \mathrm{deg}$ to the right and another $45 \mathrm{deg}$ to the left of the listener's chair. Each speaker was calibrated separately to find the signal intensity that produced the same sound pressure at the approximate location occupied by the child's head, using a sound level meter and a 1/2-in. microphone (Bruel \& Kjaer, Type 2209). (Measurements at the location of an adult's head deviated from these calibrations by less than $.5 \mathrm{~dB}$.) During calibration, the head of the microphone was pointed at the speaker being calibrated. The two levels of broad-band masking noise (42 and $60 \mathrm{dBC}$ ) were measured with the background noise being produced over both speakers at the same time and with the microphone head oriented as before.

Directly on top of each speaker, at a height of about $1.02 \mathrm{~m}$, was a plywood and smoked-glass enclosure $(61 \times 31 \times 46 \mathrm{~cm})$ which contained the toys that served as reinforcers. The glass side of this enclosure $(61 \times 46 \mathrm{~cm})$ faced the infant. During reinforcement periods, a light inside the enclosure illuminated an animated toy, which was then activated for $4 \mathrm{sec}$. A Pluto dog was used during the first test session, and a toy monkey was used for the second.

\section{Procedure}

During an experimental session, both the mother, seated on the test chair, and the infant, placed on her lap, were facing an experimenter seated in the opposite corner of the booth. An assistant remained outside the booth to adjust the attenuation levels and to record responses. Both the mother and the experimenter wore headphones over which masking noise was presented to prevent them from detecting the presence of a test signal. A trial was initiated only when the infant was quiet and was looking directly ahead, at which time the experimenter in the booth pressed a button to initiate a trial. An octave-band signal was then presented on one of the two speakers and remained on until the in- fant made a head turn of $45 \mathrm{deg}$ or more toward either side. The experimenter pressed one of two buttons to indicate the direction of the head turn. If the head turn was in the direction of the speaker carrying the signal, the signal was turned off and the toy above that speaker was illuminated and activated for a period of $4 \mathrm{sec}$. If the head turn was directed away from that speaker, the signal was turned off and there was a 4-sec intertrial interval.

To insure that infants could perform the task of turning to the sound location, they were required to meet a training criterion with sound intensity well above threshold. Initially, the signals were presented at an intensity of approximately $67.5 \mathrm{~dB}$ for the $12-, 18-$, and 24-month-old groups. During the training period, the location of the sound was alternated between left and right speakers until the infant had made four successive correct responses. The intensity was then reduced $5 \mathrm{~dB}$, and the alternation continued until the infant again made four successive correct responses. When this criterion was reached, the actual test series began. A different masking level was employed in the second session. The masking level to be used ( 42 or $60 \mathrm{dBC}$ ) in the first session was determined randomly.

During the test session, five different levels of the signal were presented a total of five times each. The randomization of sound levels consisted of five random permutations of the five levels presented sequentially. On any particular trial, the signal had a .5 chance of appearing at either speaker location with the additional constraints that the signal appear on the same speaker no more than three times in a row and appear an approximately equal number of times on the left and right. These constraints on the random order were imposed to minimize the occurrence of a response bias. With the $42-\mathrm{dB}$ masker, the signal was presented at $27.5,32.5,37.5,42.5$, and $62.5 \mathrm{~dB}$ (measured linearly with no weighting network); with the 60 -dB masker, the signal was presented at $42.5,47.5,52.5,57.5$, and $62.5 \mathrm{~dB}$ for the 12-, 18-, and 24-month-old groups. The 62.5-dB intensity, which was equivalent to the second training intensity, was used as a check on attention during the course of testing. On the basis of pilot testing, the 6-month-olds were trained and tested with intensity levels that were $5 \mathrm{~dB}$ higher than those used for the older infants. Adults were trained with the same levels as the older infants but were tested with levels that would appropriately bracket their threshold.

At the conclusion of the first session there was a rest period during which the monkey was substituted for the Pluto dog and the masking level was changed appropriately. At the beginning of the second session, the training criterion was again introduced, but this time only two correct responses at each level were required.

Twenty adult subjects were tested with the $4,000-\mathrm{Hz}$ octaveband noise at the two levels of masking noise. In a second test session, usually conducted within 2-3 days of the first session, the same adults were tested at the same two masking leveis with a $4,000-\mathrm{Hz}$ pure tone as the signal. For the pure-tone condition, the apparatus remained the same except that an oscillator (HewlettPackard, Model 204C) replaced the filtered noise source as the signal generator. To determine the sound pressure level of the pure tone, the following procedure was employed. First, we found the RMS voltage level across the speaker that produced the desired sound pressure level for a 1/3-octave band of noise centered at $4,000 \mathrm{~Hz}$. It was assumed that when the RMS voltage of the $4,000-\mathrm{Hz}$ tone was the same as the RMS voltage of the $1 / 3$ octave noise at $4,000 \mathrm{~Hz}$, the sound pressures would be approximately equal. (The sound pressure levels of the pure tone could not be determined directly because of the existence of standing wave patterns in the chamber.) For these 20 adults, procedures were identical to those for infants except that adults were directed to point to the perceived sound location, different test intensities were used, and reinforcement was limited to a light of 4-sec duration.

Thresholds for the $4,000-\mathrm{Hz}$ octave-band noise in quiet were determined for an additional group of 20 adults using two different testing procedures. In the first session, threshold was assessed during the two-alternative, forced-choice localization task 
described above. In the second session, only one speaker (either the left or right) carried the signal, and the task was to choose in which one of two intervals the signal was present. For this session, each trial was initiated by a $1-\mathrm{sec}$ warning light followed by a .5-sec pause. Two .5-sec intervals were then marked by a second light, with a .6-sec pause between intervals. The subjects, who were alone in the booth, signaled their choice of interval by pressing a button. A light of 1-sec duration signaled correct decisions. As before, the method of constant stimuli was used, and subjects were required to pass a training criterion before testing proceeded. The order of the test sessions and the speaker location in the twointerval, forced-choice task were counterbalanced across subjects.

\section{RESULTS}

\section{Thresholds in Noise}

A check on systematic order effects was made using the procedure described in Trehub et al. (1980) for those subjects who successfully completed both experimental sessions. Since there were no significant order effects ( $p>.10$ in all cases), the results for a particular masking level were collapsed over order of testing. In addition, results from infant subjects completing only a single session were included in the remaining analyses. The number of subjects at each masking level and age group ranged from 20 to 31 . Figure 2 displays the percentage of correct responses as a function of the decibel level of the octave-band signal for the two background noise levels. The parameter of the psychometric function is the age of the test group. Each point is based on a minimum of 100 trials. The psychometric functions are somewhat similar except that the infant functions are shifted 10-20 dB higher than the adult functions. At the highest intensity in the 42- $\mathrm{dB}$ masking condition, correct responding was above $95 \%$ for all groups except the 6-month-olds, whose performance was $88 \%$ correct.

The psychometric functions can be used to determine threshold values for each age group at the two masking levels. Threshold was defined as that inten-

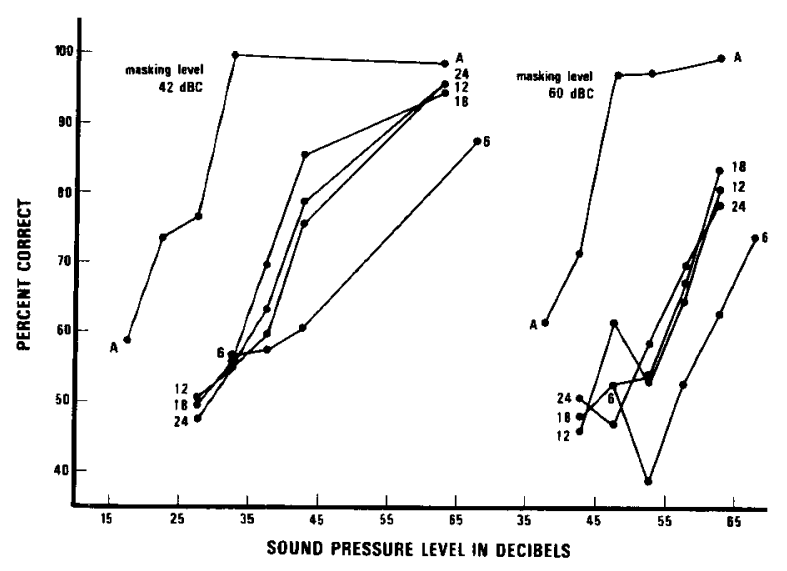

Figure 2. Percentage of correct localization responses as a functon of the decibel level of octave-band noise under two masking conditions for infants, $6,12,18$, and 24 months of age, and adults.

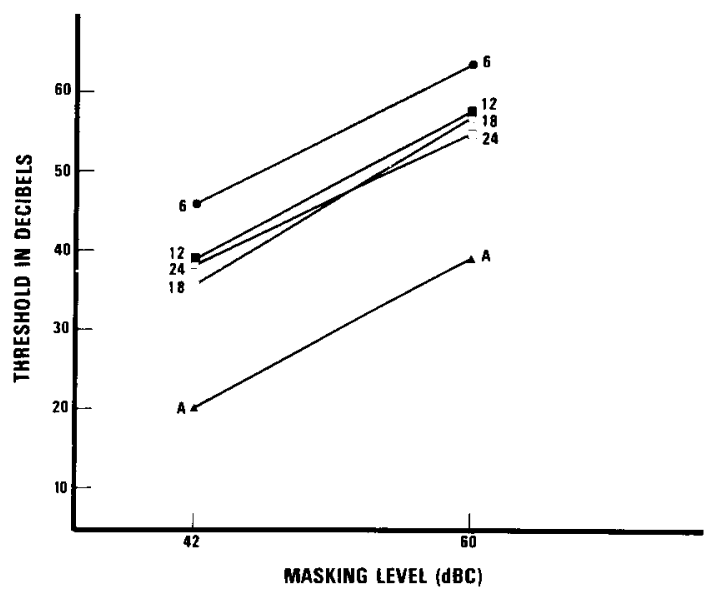

Figure 3. Thresholds as a function of masking level for infants, $6,12,18$, and 24 months of age, and adults.

sity level at which the signal was detected $65 \%$ of the time. This criterion was chosen for two reasons. First, since each point is based on a minimum of 100 trials, the probability of a sound's being correctly detected $65 \%$ of the time by chance alone is less than .002 . (In a forced-choice procedure, if the sound is inaudible, the probability of a correct response is .5. With this probability of correct responding, the normal approximation to the binomial distribution reveals a probability of equaling or exceeding $65 \%$ correct of less than .002 . Thus, an intensity level that yields $65 \%$ correct responding is clearly audible.) Second, spontaneous head turns due to inattention will have less of an effect on our estimate of stimulus detectability at lower than at higher intensity levels (Trehub et al., 1980). Consequently, we have used the $65 \%$ level as our definition of threshold.

Figure 3 shows the threshold values for the two masking conditions at the five age levels. Thresholds appear similar for 12-, 18-, and 24-month-old infants at both levels of background noise, but are approximately 7-8 dB higher for the 6-month-old group. The infant groups are considerably less sensitive (16 to $25 \mathrm{~dB}$ ) than the adults. However, for all age levels, the effect of increasing the masker by $18 \mathrm{~dB}$ is to raise the thresholds approximately $18 \mathrm{~dB}$ (an average of $18.6 \mathrm{~dB}$ for the infants and $19 \mathrm{~dB}$ for the adults).

Similar results were obtained from the same adults using a 4,000-Hz tone in the same two levels of masking noise. Figure 4 presents the adult psychometric functions for the detection of a $4,000-\mathrm{Hz}$ pure tone in the two levels of masking noise along with the adult psychometric functions from Figure 3 (detection of $4,000-\mathrm{Hz}$ octave-band noise in the same two levels of masking noise). The pure-tone thresholds are within $1.5 \mathrm{~dB}$ of those obtained with the noise band. The increase in masking level raised the threshold for the $4,000-\mathrm{Hz}$ tone by $17.25 \mathrm{~dB}$. 


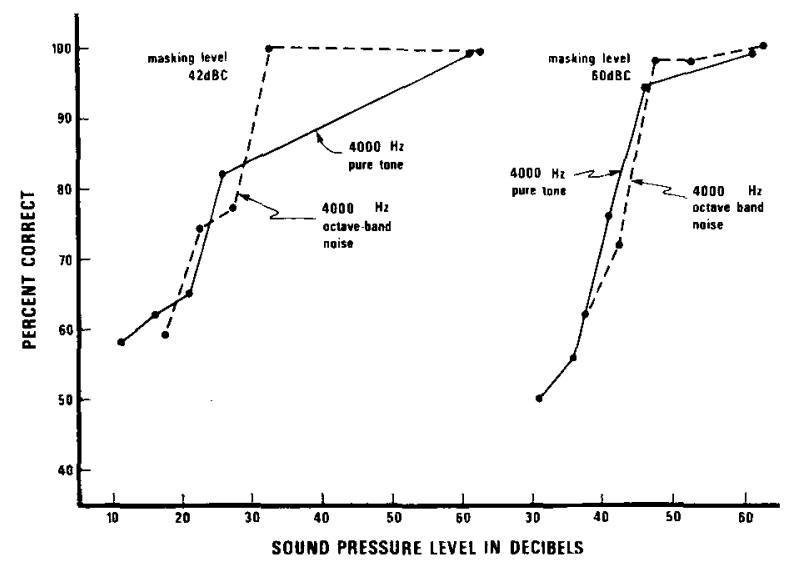

Figure 4. Percentage of correct localization responses by adults a a function of the decibel level of an octare-band noise or a tone under two masking conditions.

\section{Thresholds in Quiet}

Thresholds for the 4,000-Hz octave-band noise in quiet were also determined for an additional group of 20 adults using our localization task and a more conventional two-interval, forced-choice procedure. Since we could not detect any difference in performance due to the order in which individual subjects experienced these two tasks (using the procedures described in Trehub et al., 1980), we have collapsed over order. The psychometric functions obtained using these two tasks are shown in Figure 5. The percentage of correct responses to the five intensity levels of the 4,000-Hz octave-band noise are essentially equivalent in the two procedures. Based on a $65 \%$ criterion, the threshold values in the two tasks are equivalent $(-7 \mathrm{~dB})$.

\section{DISCUSSION}

Increasing the broad-band masking noise by $18 \mathrm{~dB}$ raised thresholds for a 4,000-Hz octave-band noise by a comparable amount for infants and adults. This is consistent with Hawkins and Stevens' (1950) classic finding that an $x \mathrm{~dB}$ increase in adults' masked threshold occurs when the masking noise is increased by $x \mathrm{~dB}$. Thus, infants and adults respond similarly to changes in the level of the masker.

However, thresholds for infants were from 16 to $25 \mathrm{~dB}$ higher than those for adults at all masking levels. A comparable adult-infant difference also occurs in the absence of masking noise. In Trehub et al. (1980), thresholds for a $4,000-\mathrm{Hz}$ octave-band noise in quiet ranged from 15 to $19 \mathrm{~dB}$ for infants 6,12 , and 18 months of age. In the present study, the mean adult threshold for the same stimulus (see Figure 5) was $-7 \mathrm{~dB}$, yielding an adult-infant difference of at least $22 \mathrm{~dB}$. Hence, there appears to be a difference of roughly $20 \mathrm{~dB}$ between adults and infants, which is more or less independent of the level of background noise.

It is always possible, of course, that the obtained adult-infant differences result from procedural and/ or motivational differences. We consider this unlikely for three reasons. First, procedural differences were minor, relating primarily to age-appropriate reinforcement. Second, a comparison of our twoalternative, forced-choice procedure with the more typical two-interval, forced-choice task yielded comparable results for adults (see Figure 5). Furthermore, the adult threshold for the $4,000-\mathrm{Hz}$ octaveband noise $(-7 \mathrm{~dB})$ was comparable to the value obtained by Robinson and Whittle (1964) using a method of limits $(-4 \mathrm{~dB})$. Hence, it is unlikely that the adult-infant differences in the present study were due to some peculiarity of our psychophysical task. Finally, if the higher thresholds for infants were due simply to motivational factors, we would expect infants to have higher thresholds in all studies using comparable techniques. Since Schneider et al. (1980) and Trehub et al. (1980) have shown that adultinfant differences in quiet depend on frequency and actually disappear at very high frequencies (around $20,000 \mathrm{~Hz}$ ), it seems reasonable to attribute the present adult-infant differences in threshold to sensory and perceptual factors as opposed to problems in motivation.

The present findings have implications for current theoretical views of masking. Neural models of the detection of signals in noise (e.g., Zwislocki, 1978) typically represent detection as depending on the internal signal-to-noise ratio. According to this position, a signal is detected when the ratio of the neural effect of the signal to the neural effect of the masker is constant. Since the detection of a signal in quiet is not perfect, it is necessary in such a model to assume the existence of some intrinsic background



Figure 5. Percentage of correct localization responses by adults as a function of the decibel level of octave-band noise and contrasting psychophysical procedures. 
noise. Thus, the threshold for a signal in quiet reflects the detection of the signal against this intrinsic background noise and presumably occurs when the ratio of the neural effect of the signal to the intrinsic background noise reaches the specified or criterial signal-to-noise ratio. In general, then, the signal is detected when

$$
\mathrm{f}(\mathrm{S}) /[\mathrm{g}(\mathrm{N})+\mathrm{b}]=\mathrm{c},
$$

where $f(S)$ is the internal effect of the signal, $g(N)$ is the internal effect of the noise, and $b$ is the amount of background neural activity in quiet. If we assume that $f$ and $g$ are both identical power functions of sound intensity, we would have

$$
\mathrm{I}_{\mathrm{s}}^{\mathrm{n}} /\left(\mathrm{I}_{\mathrm{N}}^{\mathrm{n}}+\mathrm{b}\right)=\mathrm{c}
$$

where $I_{S}$ and $I_{N}$ are the signal and noise intensities, respectively, and $n$ is a constant. Note that when the background level is sufficiently high, detection will occur when the signal-to-noise ratio in the physical domain is approximately constant, for, in that instance,

$$
\left(\mathrm{I}_{\mathrm{S}}^{\mathrm{n}} / \mathrm{I}_{\mathrm{N}}^{\mathrm{n}}\right) \cong \mathrm{c} \text {. }
$$

This model is, of course, in accord with the Hawkins and Stevens (1950) finding that an $\mathrm{x} d B$ increase in masking noise results in an $\mathrm{x} \mathrm{dB}$ increase in masked threshold.

It is interesting to note that, according to this model, adult-infant differences observed in a quiet background could result from two sources. Infants could have a higher background noise level than adults (larger b), or they could require a greater signal-to-noise ratio for detection (larger c). Finally, both $b$ and $c$ could be larger for infants. On the basis of the present infant-masking data, we can argue strongly against the first possibility. If the adultinfant threshold difference in quiet were simply due to a higher background noise, then Equation 3 would indicate that the adult-infant difference would disappear when high levels of masking noise were employed. ${ }^{1}$ But there is no suggestion in Figure 4 that adult-infant differences are reduced at the higher masking levels. If the adult-infant differences were truly independent of masking level, we could then conclude safely that the data supported the notion that adults and infants had the same level of internal background noise but that infants required a higher internal signal-to-noise ratio for detection. The present data are somewhat inconclusive on this point. A comparison of the present study and Trehub et al. (1980) indicates that the adult-infant difference in quiet might be somewhat larger than that in background noise. This would be consistent with the notion that both the background noise level and the signal-to-noise ratio required for detection are larger for infants than for adults. However, since the comparison was across studies using different subjects, we cannot decide at present whether the adult-infant difference is due simply to a larger signal-to-noise ratio at threshold or to both a larger signal-to-noise ratio and a higher background level.

It is also worth noting that the data are not consistent with what would be expected if infants were simply subject to a conductive hearing loss. Suppose infants experienced a $20-\mathrm{dB}$ conductive hearing loss as compared with adults. In a quiet background (noise intensity equal to zero), Equation 2 would indicate that the signal intensity for infants would have to be $20 \mathrm{~dB}$ higher than for adults in order to arrive at the same value of $c$. However, for the detection of signals in very noisy backgrounds, where Equation 3 holds, there should be no adult-infant difference. Equation 3 shows that at high levels of masking, noise threshold is reached when the physical signal-to-noise ratio is constant. A conductive hearing loss would attenuate both signal and noise intensities, leaving the effective signal-to-noise ratio unchanged. Consequently, if adult-infant differences at threshold are to be attributed solely to a conductive hearing loss, then these threshold differences should disappear at high levels of background noise. Figure 4 shows that they clearly do not. In any event, Hecox (1975) has argued that anatomical immaturity in infancy could account for no more than a 10-dB conductive loss in sensitivity.

On a purely practical level, the observed differences in masked threshold represent a potential impairment of substantial magnitude for the infant groups. The 6-month-old group, for example, required a signal $20 \mathrm{~dB}$ greater than that required by adults for detection in the same level of background noise. Discrepancies between adults and infants have also been reported by Trehub, Bull, and Schneider (1981) for detection of a speech phrase presented in the same levels of background noise as the present study. Using the same two-alternative, forced-choice technique, the difference between infant and adult masked thresholds was approximately $10 \mathrm{~dB}$. Since adult-infant differences are smaller for some frequencies than for others, this discrepancy does not, in theory, pose a problem. A speech signal has its energy spread over a broad frequency range, and the detection of such a signal could depend on its most detectable component. Furthermore, the calibration of the speech signal in Trehub et al. (1981) was with respect to its average intensity. The detection of speech in noise will depend, then, on the spectral composition of the signal among other things.

Our results and those of Trehub et al. (1981) raise the possibility that in a moderately noisy environment infants may not perceive some signals that are audible for adults. While Mills (1975) has argued that 
current levels of environmental noise may interfere with speech, language, and listening skills in children, evidence in support of this claim is currently insufficient. The relative masking impairment of infants as shown in the present study suggests that the acquisition of communication skills may be impeded in very noise environments.

\section{REFERENCES}

Elliott, L. L., \& Katz, D. R. Children's pure-tone detection. Journal of the Acoustical Society of America, 1980, 67, 343-344.

Hawkins, J. E., \& Stevens, S. S. The masking of pure tones and of speech by white noise. Journal of the Acoustical Society of America, 1950, 22, 6-13.

Hecox, K. Electrophysiological correlates of human auditory development. In L. B. Cohen \& P. Salapatek (Eds.), Infant perception: From sensation to cognition (Vol. 2). New York: Academic Press, 1975.

Mills, J. H. Noise and children: A review of literature. Journal of the Acoustical Society of America, 1975, 58, 767-779.

Moore, J. M., \& WiLson, W. R. Visual reinforcement audiometry (VRA) with infants. In S. E. Gerber \& G. T. Mencher (Eds.), Early diagnosis of hearing loss. New York: Grune \& Stratton, 1978.

Moore, J. M., Wilson, W. R., \& Thompson, G. Visual reinforcement of head-turn responses in infants under twelve months of age. Journal of Speech and Hearing Disorders, 1977, 42, 328-334.

Robinson, D. W., \& Whitrte, L. S. The loudness of octavebands of noise. Acustica, 1964, 14, 24-35.

Schneider, B. A., TrehuB, S. E., \& Bull, D. High-frequency sensitivity in infants. Science, 1980, 207, 1003-1004.

Trenub, S. E., Bull, D., \& Schneider, B. A. Infants' detection of speech in noise. Journal of Speech and Hearing Research, 1981, 24, 202-206.

Trehur, S. E., Schneider, B. A., \& Bull, D. The effect of reinforcement on infants' performance in an auditory detection task. Developmental Psychology, 1981, 17, in press.

Trehub, S. E., Schneider, B. A., \& Endman, M. Developmental changes in infants' sensitivity to octave-band noises. Journal of Experimental Child Psychology, 1980, 29, 282-293.

ZwisLockI, J. J. Masking: Experimental and theoretical aspects of simultaneous, forward, backward, and central masking. In E. C. Carterette \& M. P. Friedman (Eds.), Handbook of perception (Vol. 4) Hearing. New York: Academic Press, 1978.

\section{NOTE}

1. To see that this is indeed the case, let $I_{t}$ be the threshold intensity for adults in quiet. Then, from Equation 2, we compute that the amount of background neural activity in quiet for adults is $b=I_{t}^{n} / c$. Suppose the threshold in quiet is $20 \mathrm{~dB}$ higher for children than it is for adults. Then the threshold intensity for children is 100 times that of adults ( $20 \mathrm{~dB}$ is a $100 / 1$ intensity ratio) and the level of background neural activity for children is $b=$ $(100 \mathrm{I})^{\mathrm{n}} / \mathrm{c}$. Suppose, further, that the background noise is $60 \mathrm{~dB}$ higher than the threshold intensity for adults, that is, $I_{N}=10^{6} I_{t}$. Using Equation 2, we can now compute the signal intensity necessary to reach threshold in this background of $60 \mathrm{~dB}$ noise for both adults and children. For adults,

$$
I_{s}=\left\{c\left[\left(10^{6} I_{t}\right)^{n}+\left(I_{t}^{n}\right) / c\right]\right\}^{1 / n} .
$$

The corresponding intensity for children is

$$
I_{s}=\left\{c\left[\left(10^{6} I_{t}\right)^{n}+\left(100 I_{t}\right)^{n} / c\right]\right\}^{1 / n} \text {. }
$$

Therefore, the decibel difference between the masked threshold for adults and children is 10 times the logarithm of the ratio of the masked threshold intensity for adults to the masked threshold intensity for children. This decibel difference works out to be

$$
10 / n \log \left[\left(10^{6 n}+1 / c\right) /\left(10^{6 n}+100^{n} / c\right)\right] .
$$

It is clear that the larger the value of $c$ (the signal-to-noise ratio), the smaller the threshold difference. To arrive at a conservative estimate of the largest difference in masked thresholds that we might expect to find, we set $c=1$. (It is unlikely that the signalto-noise ratio at threshold is smaller than this value.) With our masking stimulus $60 \mathrm{~dB}$ higher than the adult threshold and with the exponent of the power function equal to .3 , we would expect the adult masked threshold to be only $.66 \mathrm{~dB}$ lower than the masked threshold for infants. In the present study, the highest level of the masking stimulus is approximately $67 \mathrm{~dB}$ above the threshold for the signal in quiet. Even if only $1 / 4$ of the total power in this masking stimulus was in the critical band around $4,000 \mathrm{~Hz}$, the effective masking signal would be greater than $60 \mathrm{~dB}$ above the absolute threshold for adults. Clearly, then, the threshold differences observed in quiet between adults and infants cannot be due solely to a higher degree of physiological noise for infants.

(Manuscript received March 26, 1981; revision accepted for publication May 26, 1981.) 